\title{
USO DO MODELO WEATHER RESEARCH AND FORECASTING (WRF) PARA CIRCULAÇÃO SUPERFICIAL EM FORTALEZA DURANTE O PROJETO CHUVA
}

\author{
Vinícius Milanez Couto ${ }^{1,}$, Natanael Vieira de Sousa ${ }^{1}$, João Bosco Verçosa Leal Junior ${ }^{1}$. \\ ${ }^{1}$ Universidade Estadual do Ceará (UECE) \\ *vimcless@gmail.com
}

\section{RESUMO}

O objetivo deste trabalho é o de analisar a velocidade e a direção do vento utilizando o modelo atmosférico Weather Research and Forecasting, para dois esquemas físicos de Camada Limite diferentes. Observou-se que as simulações representaram de forma satisfatória as duas variáveis.

\begin{abstract}
This work aims to analyse de wind speed and direction using the Weather and Research Forecasting atmospheric model, with 2 different Boundary Layer parameterization. The two simulations represented satisfactorily the two atmospheric variables, but the wind speed was overestimated.
\end{abstract}

\section{INTRODUÇÃO}

É cada vez mais comum o uso de modelos atmosféricos computacionais para se analisar os fenômenos ou para previsão do tempo. Mas para se fazer uma boa análise da atmosfera através de modelos, é necessário compara-lo com dados observados.

Durante o mês de abril de 2011 houve em Fortaleza a primeira etapa do Projeto Chuva, cujo objetivo era o de armazenar os dados e utiliza-los para melhor compreender os principais sistemas precipitantes do país. 
O objetivo deste trabalho é avaliar o modelo atmosférico Weather Research and Forecasting (WRF) para as variáveis velocidade e direção do vento e comparar a influência do esquema de Camada Limite nestas variáveis.

\section{MATERIAIS E MÉTODOS}

Obtiveram-se os dados de velocidade e direção do vento a 10 metros de altura para o período do dia 15 até o dia de 22 de abril de 2011. Os dados, que inicialmente estão em média a cada minuto, foram então retirados apenas a cada 10 minutos que coincidem com os dados modelados.

O modelo computacional utilizado é o Weather Research and Forecasting (SKAMAROCK;KLEMP, 2008), versão 3.3. As configurações foram: 4 grades, com centro nas coordenadas da Plataforma de Coleta de Dados instalada na Defesa Civil de Fortaleza $\left(3,729^{\circ} \mathrm{S} 38,564^{\circ} \mathrm{O}\right)$, espaçamento de grade de $27 \mathrm{~km}, 9 \mathrm{~km}, 3 \mathrm{~km}$ e $1 \mathrm{~km} .56,94,124$ e 184 pontos de grade, 51 níveis verticais, com topo do modelo de $70 \mathrm{hPA}$, dados de entrada e de contorno a cada 6 horas do FNL, esquemas físicos RRTMG para as radiações de ondas de onda longa e curta, WDM6 para microfísica, NSAS para cumulus nas duas grade mais grossas. Para ver a influência do esquema físico da Camada Limite, efetuaram-se duas simulações, uma utilizando o esquema ACM2, outra com o esquema MYJ.

Para avaliar o modelo, utilizou-se o viés $(b)$, erro absoluto médio $(M A E)$, raiz do erro quadrático médio (RMSE), correlação de Pearson ( $r$ ), índice de concordância refinado $\left(d_{r}\right)$ e o índice de eficiência modificado $\left(E_{l}\right)$ (LEGATES; MCCABE, 1999; MORIASI et al., 2007; WILLMOTT; ROBESON; MATSUURA, 2012).

\section{RESULTADOS E DISCUSSÃO}

Estatisticamente, houve boa concordância entre os modelos e os dados observados: $r=0,73$ e $0,76, \mathrm{~d}_{\mathrm{r}}=0,51$ e $0,63, \mathrm{E}_{1}=0,03$ e $0,26, \mathrm{~b}=1$ e $0,54, \mathrm{MAE}=1,22$ e 0,93 e $\mathrm{RMSE}=1,44$ e 1,13 para a simulação ACM2 e MYJ, respectivamente, para a variável velocidade do vento. Para a direção, $r=0,54$ e $0,57, \mathrm{~d}_{\mathrm{r}}=0,58$ e $0,58, \mathrm{E}_{1}=0,16$ e $0,17, \mathrm{~b}=-17$ e $-16, \mathrm{MAE}=26$ e 26 e $\mathrm{RMSE}=38$ e 37. 
Pode ser visto na Figura 1 que para as duas simulações, houve uma tendência a seguir o ciclo diário, mesmo existindo uma superestimativa para a magnitude do vento, principalmente para o ACM2.

Na Figura 2, é observado que a predominância do vento é bem representada, mas com uma maior frequência, ou seja, menor variação na direção (menor de 70\%).
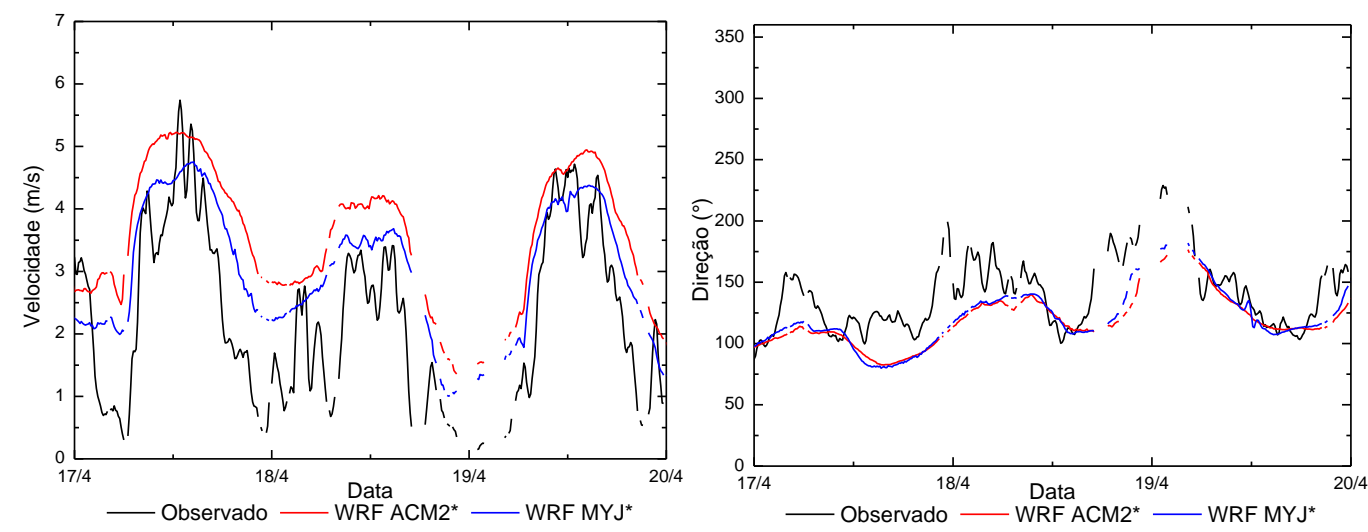

Figura 1: Série temporal da velocidade (esquerda) e direção (direita) do vento a 10 metros de altura durante entre os dias 17 e 20 de abril de 2011.

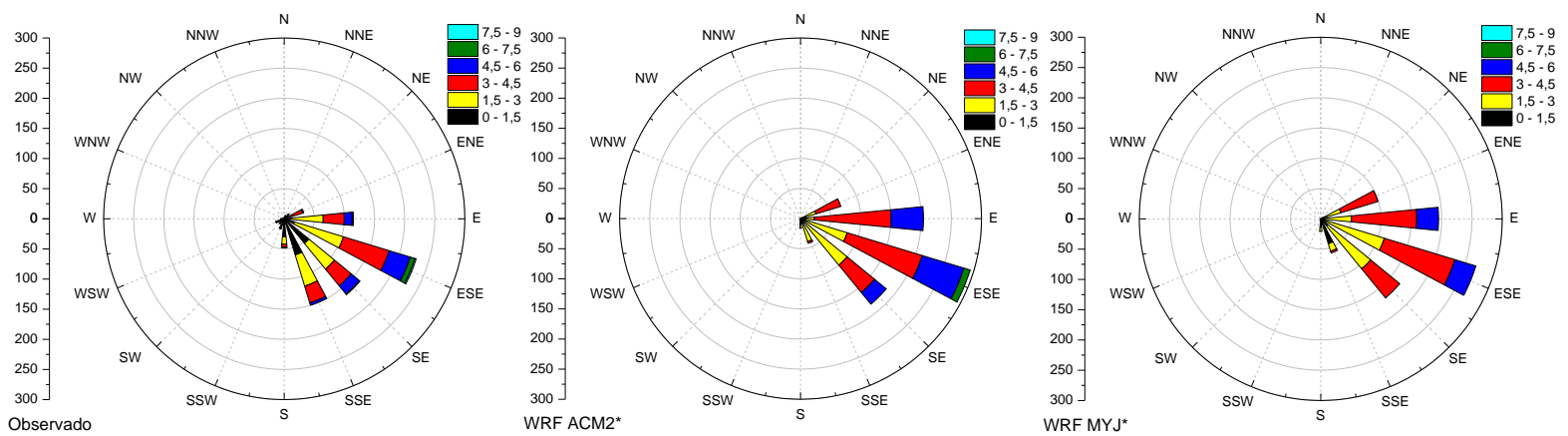

Figura 2: Rosa dos ventos para a altura de 10 metros durante o período estudado.

\section{CONCLUSÕES}

Houve boa concordância entre os dados de velocidade e direção do vento. Houve uma superestimação na velocidade de $1 \mathrm{~m} / \mathrm{s}$ e $0,54 \mathrm{~m} / \mathrm{s}$ para a simulação ACM2 e MYJ, respectivamente. A direção predominante condiz com os dados observados, mas a variação do vento foi suavizada. 


\section{AGRADECIMENTOS}

A CAPES e FUNCAP pelo apoio financeiro e ao Projeto Chuva (FAPESP 2009/15235-8) pela cessão dos dados.

\section{REFERÊNCIAS BIBLIOGRÁFICAS}

LEGATES, D. R., AND G. J. MCCABE JR. , Evaluating the use of "goodness-of-fit" Measures in hydrologic and hydroclimatic model validation, Water Resour. Res., V. 35, No 1, p. 233-241, doi:10.1029/1998WR900018. 1999.

MORIASI, D.; ARNOLD, J.; V. L., M.; BINGNER, R.; HARMEL, R.; VEITH, T. Model evaluation guidelines for systematic quantification of accuracy in watershed simulations. Transactions of the American Society of Agricultural and Biological Engineers, v. 50, n. 3, p. 885-900, 2007.

SKAMAROCK, W. C.; KLEMP, J. B. A time-split nonhydrostatic atmospheric model for weather research and forecasting applications. Journal of Computational Physics, V. 227, $\mathrm{N}^{\mathbf{}}$ 7, p. 3465-3485, ISSN 0021-9991, http://dx.doi.org/10.1016/i.jcp.2007.01.037. 2008.

WILLMOTT, C. J., ROBESON, S. M. AND MATSUURA, K., A refined index of model performance. Int. J. Climatol. V. 32, No, p. 2088-2094. doi: 10.1002/joc.2419. 2012. 